\title{
AVALIAÇÃO DA FUNCIONALIDADE DO MEMBRO SUPERIOR PARÉTICO DE PACIENTES COM SEQUELA DE AVE APÓS PROTOCOLO TERAPIA POR CONTENSÃO INDUZIDA
}

\author{
Débora Benfeita de Souza Amaral', Mariana da Silva Gomes', Izadora Dias Coutinho', Daniele \\ Azevedo Ramos', Neydimara Viana Gomes, Thais Barbosa Pereira ${ }^{1}$, Winny da Silva Gomes 1 , \\ Thayane Henriques Lemos', Kaciane Lopes Ferreira', Marcelle Barreto de Abreu', Camila \\ Gonçalves Santana $^{2}$ \& Elizabeth Viana Soares ${ }^{2 *}$
}

\begin{abstract}
RESUMO
AMARAL, D.B.S.; GOMES, M.S.; COUTINHO, I.D.; RAMOS, D.A.; GOMES, N.V.; PEREIRA, T.B.; GOMES, W.S.; LEMOS, T.H.; FERREIRA, K.L.; ABREU, M.B.; SANTANA, C.G. \& SOARES, E.V. Avaliação da funcionalidade do membro superior parético de pacientes com sequela de ave após protocolo terapia por contensão induzida. Perspectivas Online: Biológicas e Saúde, v.7, n.24, p.81-87, 2017.

Introdução: A limitação para realizar as funções com o membro superior parético após um AVC é uma das maiores queixas dos pacientes após a lesão. Objetivo: verificar se a técnica de terapia por contensão induzida promove melhor funcionalidade ao membro superior. Metodologia: Foi realizado um estudo observacional descritivo do tipo não participativo, na Clínica Escola Maria Auxiliadora, com pacientes paréticos MS após AVC que foram submetidos por 2 semanas consecutivas, com uma frequência $5 \mathrm{x} /$ semana por 3horas a TCI. Os pacientes foram avaliados para a realização das funções de levar a mão a boca e a axila contralateral, onde foram mensurados a

distância e o tempo de execução da função nos períodos pré e pós tratamento, e cálculo da velocidade média. Resultados: A amostra foi composta por 2 pacientes, de ambos os sexos, com lesão no dimidio direito. Houve na análise dos resultados um aumento na velocidade média de $15,05 \mathrm{~m} / \mathrm{s}$ para $29,05 \mathrm{~m} / \mathrm{s}$ e diminuição no tempo para realização das tarefas de $1,5 \mathrm{~s}$ para $0,72 \mathrm{~s}$ nos momentos pré e pós. Conclusão: foi possível observar que a TCI influenciou positivamente na realização da função motora do membro superior parético pós AVE, contribuindo para melhora na velocidade média e diminuição no tempo de execução das tarefas.
\end{abstract}

Palavras Chaves: Terapia por Contensão Induzida, Funcionalidade, Paresia. 


\begin{abstract}
Introduction: The limitation to performing the functions with the paretic upper limb after a stroke is one of the biggest complaints of patients after the injury. Objective: To verify if the technique of induced contention therapy promotes better functionality to the upper limb. Methods: A descriptive, non-participatory observational study was performed at the Maria Auxiliadora School Clinic with MS patients after stroke who were submitted for 2 consecutive weeks at a frequency of $5 \mathrm{x} /$ week for 3 hours at ICU. The patients were evaluated for performing the functions of hand to mouth and contralateral armpit, where distance

and time of function execution were measured in the pre and post treatment periods, and mean velocity calculation. Results: The sample consisted of 2 patients of both sexes, with lesion in the right dimidium. There was an increase in the mean velocity from $15.05 \mathrm{~m} / \mathrm{s}$ to $29.05 \mathrm{~m} / \mathrm{s}$ and a decrease in the time to perform the tasks from $1.5 \mathrm{~s}$ to $0.72 \mathrm{~s}$ in the pre and post moments. Conclusion: it was possible to observe that the TCI positively influenced the motor function of the paretic upper limb post AVE, contributing to an improvement in the mean velocity and a decrease in the task execution time.
\end{abstract}

Key Words: Induced Congestion Therapy, Functionality, Paresis.

\footnotetext{
${ }^{1}$ Acadêmicos em Fisioterapia do ISECENSA e colaboradores do PROVIC - Rua Salvador Correa, 139, Centro, Campos dos Goytacazes, RJ, CEP: 28035-310, Brasil

${ }^{2}$ Institutos Superiores de Ensino do CENSA - ISECENSA - Laboratório de Análise do movimento - Rua Salvador Correa, 139, Centro, Campos dos Goytacazes, RJ, CEP: 28035-310, Brasil;

(*) e-mail: elizabethvsoares@outlook.com

Data de chegada: 17/04/2017 Aceito para publicação: 19/06/2017
}

Persp. online: biol. \& saúde, Campos dos Goytacazes, 24 (7), 81-87, 2017

seer.perspectivasonline.com.br 


\section{INTRODUÇÃo}

O AVE pode ocorrer por uma falta súbita de suprimento sanguíneo gerando ainda déficit na concentração de oxigenação com consequente morte neuronal e perda das funções envolvendo o cognitivo, a parte motora e sensorial impedindo o paciente de conseguir realizar as atividades da vida diária de forma independente (ROLIM, 2011).

Segundo a Organização Mundial de Saúde, o Acidente Vascular Encefálico (AVE) é a terceira maior causa de morte no mundo depois das doenças cardíacas e do câncer. Além de ser a principal causa de incapacidade nos Estados Unidos, com aproximadamente 730.000 casos por ano (Feigin et al, 2003; Strong et al, 2007), e na América Latina, o AVE encontra-se também entre os principais problemas de saúde pública, apesar de os poucos estudos epidemiológicos existentes não quantificarem a real dimensão do problema (Lotufo, 2005; Saponisk et al, 2003). De acordo com Moura (2015), a mortalidade causada pelo AVE varia de $13,8 \%$ a $20,6 \%$ em pacientes com idade superior a 60 anos gerando também graves incapacidades.

Segundo Medeiros (2013), 80\% dos pacientes com sequela de AVE apresentam alterações físicas, emocionais e sociais que se traduzem por comprometimento sensório motor importante. Dentre as complicações mais comuns encontra-se a hemiparesia, definida como diminuição da força muscular no dimidio corporal afetado em prol de uma lesão encefálica no lado contralateral do corpo.

Associado a hemiparesia, os pacientes ainda apresentam modificação no tônus (hipertonia espástica ou hipotonia), alterações sensoriais, visuais, reflexos, força muscular e presença de dor durante o retorno dos movimentos ou mesmo em repouso, alterações nas funções do tronco prejudicando a estabilidade e mobilidade do esqueleto apendicular (PIASSAROLI, 2012).

Costa (2006) relata que, o funcionamento motor dos pacientes hemiparéticos pode sofrer alterações em virtude das alterações na força muscular, na sensibilidade, no tônus muscular e na coordenação motora, influenciando diretamente no controle postural do tronco e limitação para realização das contrações do tipo excêntrica no lado menos acometido pelo AVE.

Em virtude do AVE, os pacientes que apresentam alteração na força muscular apresentam déficits funcionais do segmento superior. A força muscular pode ser mensurada através do teste de força muscular manual (que varia de 0 a 5) ou de dinamômetro o membro superior. Os testes acima são amplamente utilizados na prática fisioterapêutica como instrumentos avaliadores para planejamento terapêutico (SALMELA, 2000)

A diminuição de força muscular associado a alteração do tônus e a presença de fenômenos adaptativos são fatores suficientes para permitir alterações funcionais no membro superior parético (SALMELA, 2000)

Sendo assim, os pacientes com paresia de membro superior não realizam a correta flexão do ombro (pelos seus músculos flexores), compensando através do uso excessivo das fibras superiores do trapézio gerando uma elevação do ombro com flexão lateral do tronco para o outro lado, devido a falta de força. Essa alteração pode ser considerada como uma das causadoras de processos dolorosos no ombro desses pacientes (TELES, 2011).

As condutas terapêuticas utilizadas atualmente são as mais diversas, incluindo o uso de recursos analgésicos, eletroterápicos, miofasciais, técnicas específicas de fortalecimento (Facilitação Neuromusculat Proprioceptiva-FNP, Bobath, cinesioterapia resistida), terapia por contensão induzida, dentre outras.

A TCI possui o objetivo de reabilitar o membro superior parético melhorando a funcionalidade, aumentando a amplitude de movimento, diminuindo a negligência do membro superior. Esta técnica é realizada num período de 5 dias, durante 2 semanas consecutivas, por 3 horas e associada a exercícios 
domiciliares. Para que a técnica seja executada é necessário o uso de uma luva para conter a mão do lado menos afetado, estimulando o uso da mão mais afetada.

Sendo assim, este projeto visa avaliar a funcionalidade do membro superior parético de pacientes que foram submetidas a técnica Terapia por Contensão Induzida.

\section{METODOLOGIA}

Foi realizado um estudo observacional descritivo do tipo não participativo, aprovado pelo Comitê de Ética dos Institutos Superiores de Ensino do ISECENSA antes da sua execução, e realizado no Laboratório de Fisioterapia Neurofuncional da Clínica Escola Maria Auxiliadora (CEMA).

A amostra foi composta por 02 pacientes hemiparéticos crônicos pós AVE (com12 meses de lesão) de ambos os sexos, com idade de 15 a 90 anos. As variáveis de interesse foram: sexo, tempo de lesão, dimidio corporal lesionado, com hemiparesia em ambos os lados que estejam sendo tratados através da técnica terapia por contensão induzida para recuperação da função do MS acometido. Foram excluídos do estudo hemiparéticos com espasticidade grave - segundo escala de Ashworth Modificada, com déficit cognitivo, em uso de drogas psicotrópicas ou psiquoativas, que apresentem crises convulsivas, com negligência hemiespacial ou apraxia e que não assinarem o termo de consentimento livre e esclarecido.

Os pacientes foram solicitados a realizar o movimento funcional de levar a mão a boca e a mão a axila contralateral a lesão, onde cronometrou-se o tempo e a distância para execução das funções. Posteriormente foi calculado a velocidade média através da fórmula $\mathrm{VM}=\Upsilon \mathrm{S} / \mathrm{XT}$ (variação da distância / variação do tempo). Esta avaliação foi realizada no pré e pós intervenção da terapia por contensão induzida para MS parético.

A aplicação da técnica ocorreu com 3 horas de intervenção diária, 5x/semana, por 2 semanas, além da utilização da luva de contensão por $90 \%$ do tempo no ambiente domiciliar durante as duas semanas de realização da técnica.

Os dados obtidos foram inseridos e organizados em planilhas do programa Microsoft Excel ${ }^{\circledR} 2016$ (Microsoft Corporation; Redmond, WA, EUA), possibilitando, análise descritiva dos resultados e a confecção dos gráficos para cada variável.

\section{RESULTADOS}

O presente estudo obteve uma amostra composta por 2 pacientes, com as seguintes caracterísiticas: paciente 1 do sexo feminino, 60 anos de idade, com 2 meses de lesão, acometimento no hemisfério cerebral direito. $\mathrm{O}$ paciente 2 é do sexo masculino, com idade 31 anos, comprometimento no hemisfério cerebral direito após lesão isquêmica.

$\mathrm{Na}$ análise da velocidade média e tempo, os voluntários submetidos a TCI mostraram melhora clínica na execução das funções levar a mão a boca e a axila contralateral. A velocidade média na execução da tarefa levar a mão a boca era de $15,05 \mathrm{~cm} / \mathrm{s}$ no pré tratamento evoluindo para $29,65 \mathrm{~cm} / \mathrm{s}$ no pós tratamento. Com o aumento da velocidade média consequentemente houve diminuição no tempo (s) de execução das funções que variou de $1,50 \mathrm{~s}$ no pré para $0,72 \mathrm{~s}$ no pós (figura 1 ).

$\mathrm{Na}$ análise da função de levar a mão na axila contralateral no pré tratamento a amostra realizou a função com velocidade média de e $46,32 \mathrm{~cm} / \mathrm{s}$ avançando para $89,43 \mathrm{~cm} / \mathrm{s}$. O tempo de execução da função diminuiu de $1,87 \mathrm{~s}$ no pré tratamento para $0,92 \mathrm{~s}$ no pós tratamento (figura 2 ). 


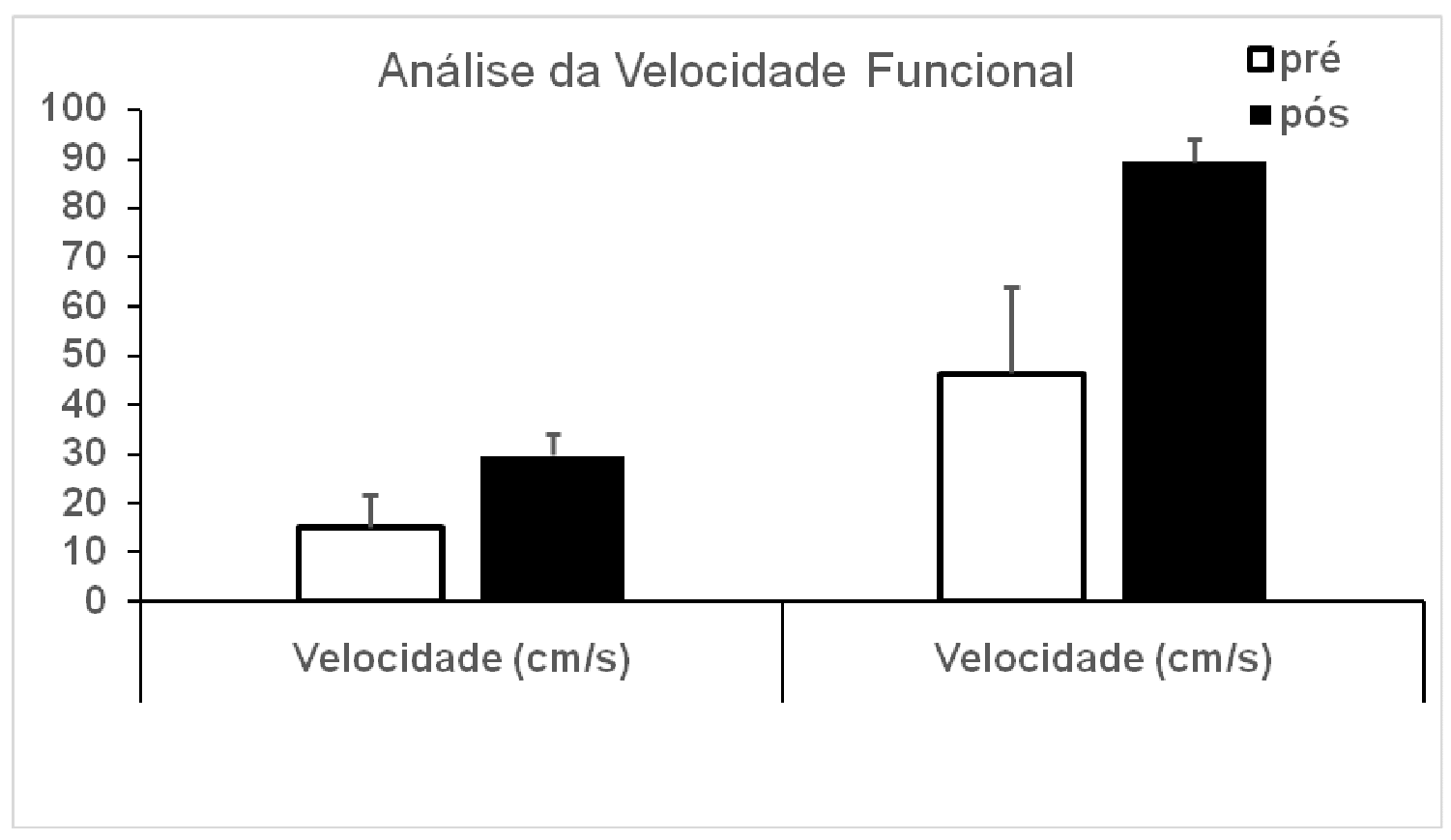

Figura 1: Média \pm DP da velocidade média das funções levar a mão a boca (esquerda) e a axila contralateral (direita) nos instantes pré (branco) e pós (preto). Não foi evidenciado diferença estatisticamente significativa $(\mathrm{p}<0,5)$.

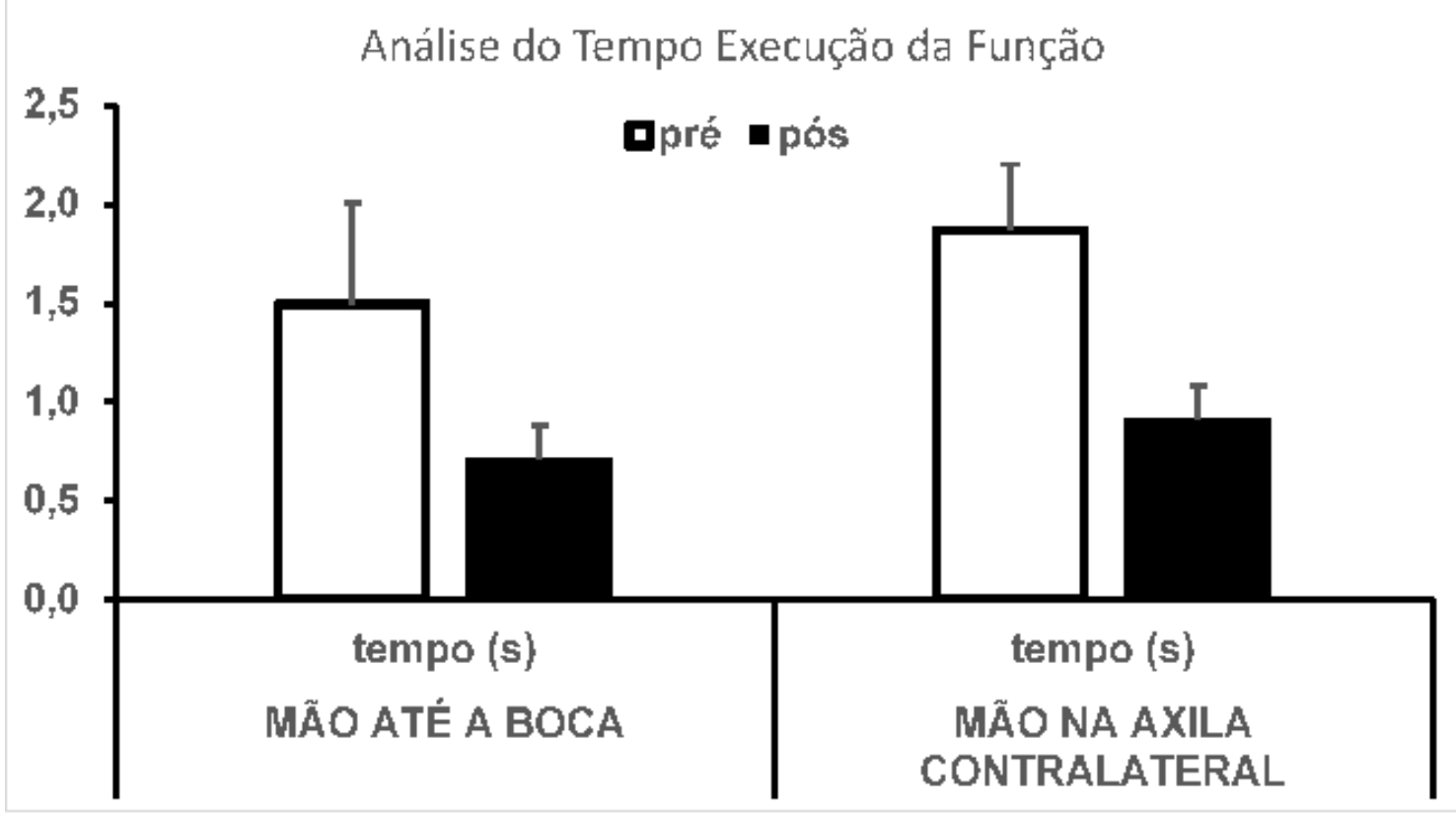

Figura 2: Média $\pm \mathrm{DP}$ do tempo em segundos (s) das funções da mão até a boca (esquerda) e a mão na axila contralateral (direita) nos instantes pré (branco) e pós (preto). Não foi observada diferença estatisticamente significativa na realização das funções nos momentos pré e pós. 


\section{DISCUSSÃo}

De acordo com dados esclarecidos anteriormente, com relação as características da amostra, observase $50 \%$ da amostra foi do sexo feminino. De acordo com Bensenor (2015), a prevalência do AVC (1,6 para homens e 1,4\% para mulheres) e das incapacidades funcionais são similares (29,5\% para homens e $21,5 \%$ para mulheres), o que corrobora com os dados encontrados neste estudo.

Segundo Meneghetti et al (201) a TCI proporciona bons resultados após a sua aplicação em relação à qualidade e funcionalidade do movimento.

Em estudo realizado por Pereira et al. (2010) concluiu-se que quando é aplicado os três componentes da TCI (restrição do segmento menos afetado, treinamento intensivo orientado à tarefa e métodos de transferência) pode ocorrer maior impacto nas atividades de vida diária (AVD'S) dos pacientes paréticos submetidos à técnica.. Pode-se então, atribuir a diminuição do tempo de execução das tarefas impostas, a melhora da função do membro superior, resultando em melhora de coordenação do movimento e força visualizado através da maior habilidade e destreza na realização das funções, segundo este autor em seu estudo.

Sant'Anna, et al. (2014) cita que partindo do princípio que a neuroplasticidade é a capacidade do sistema nervoso de modificação e adaptação a nível estrutural e funcional em reação a novas experiências, ouso de novas técnicas que induzem essa potencialização interferem na plasticidade cerebral. A aprendizagem, a prática e a repetição de determinadas atividades, favorecem as mudanças plásticas do sistema nervoso central (SNC) contribuindo assim, para a reabilitação do paciente neurológico.

\section{CONCLUSÃO}

Através dos resultados obtidos neste estudo, foi possível observar que a TCI influenciou positivamente na função motora do membro superior parético pós AVE, contribuindo para o aumento da velocidade média e a diminuição do tempo de execução das tarefas.

\section{REFERÊNCIAS}

BENSENOR, I.M; GOULART, A.C; SZWARCWALD, C.L; VIEIRA, M.L.F.P; MALTA, D.C; LOTUFO, P.A. Prevalência de acidente vascular cerebral e de incapacidade associada no Brasil: Pesquisa Nacional de Saúde - 2013

COSTA M.C.F; BEZERRA P.P; OLIVEIRA A.P.R. Impacto da hemiparesia na simetria e na transferência de peso: repercussões no desempenho funcional. REVISTA NEUROCIÊNCIAS V14 N2 - ABR/JUN, 2006 (010-013).

FEIGIN V.L; LAWES C.M; BENNETT D.A; ANDERSON C.S. Stroke epidemiology: a review of population-based studies of incidence, prevalence, and case-fatality in the late 20th century. Lancet Neurol. 2003;2(1):43-53.

LOTUFO P.A. Stroke in Brazil: a neglected disease. Sao Paulo Med J. 2005;123(1):3-4.

MEDEIROS, J.M; GRANJA, K.S.B; PINTO, A,P, S; Avaliação do impacto do acidente vascular cerebralsobre a população acometida: revisão sistemática. Cadernos de Graduação - Ciências Biológicas e da Saúde, Maceió; v. 1 n.3 p. 131-136 nov. 2013

MENEGHETTI, C. H. Z.;SILVA, J. A.; GUEDES, C. A. V.Terapia de restrição e indução ao movimento no paciente com AVC: relato de caso.RevNeurocienc 2010;18(1):18-23.

Persp. online: biol. \& saúde, Campos dos Goytacazes, 24 (7), 81-87, 2017

seer.perspectivasonline.com.br 
MOURA, M; CASUALARI, L.A. Impacto da adoção de medidas inespecíficas no tratamento do acidente vascular cerebral isquêmico em idosos: a experiência do Distrito Federal, Brasil. Rev Panam Salud Publica $38(1), 2015$

PEREIRA, N. D.; MENEZES, I. S.; ANJOS, S. M. Uso de três princípios de intervenção aumenta a efetividade da Terapia por Contensão Induzida: estudo de caso.Rev. Ter. Ocup. Univ. São Paulo, v. 21, n. 1, p. 33-40, jan./abr. 2010.

PIASSAROLI, C.A.P; ALMEID, G.C; LUVIZZOTO, J.C; SUZAN, A.B.BM. Modelos de reabilitação fisioterápica em pacientes adultos com sequelas de AVC isquêmico. Rev Neurocienc 2012;20(1):128-137

ROLIM, C.R.L.C; MARTINS, M. Qualidade do cuidado ao acidente vascular isquêmico no SUS. Cad. Saúde Pública, Rio de Janeiro, 27(11):2106-2116, nov, 2011

SALMELA, L.F.T et al. Fortalecimento Muscular e Condicionamento Físico em Hemiplégicos. Acta Fisica, dezembro 2000 , volume $7 \mathrm{n}^{\circ} 3$.

SANT'ANNA, L. F., GUIDA, S., SILVA, J.G. Informações Somatossensoriais nos Processos da Prática Mental na Fisioterapia Neurofuncional: Estudo de Revisão.Rev Neurocienc 2014;22(1):95-101.

SAPONISK G; DEL BRUTTO O.H. Stroke in South America: a systematic review of incidence, prevalence and stroke subtypes. Stroke. 2003; 34(9):2103-8.

STRONG K; MATHERS C; BONITA R. Preventing stroke: saving lives around the world. Lancet Neurol. 2007;6(2):182-7.

TELES, M.S; GUSMÃO, C. Aavaliação funcional de pacientes com acidente vascular cerebral utilizando o protocolo de Fulg-Meyer. Rev Neurocienc 2012;20(1):42-49 